\title{
AHP : The signal and the noise - A numerical experiment on the possibility of getting the solution with much less pairwise comparisons
}

Robin Rivest, M.Sc. Candidate at HEC Montreal,

Montreal, Quebec, Canada

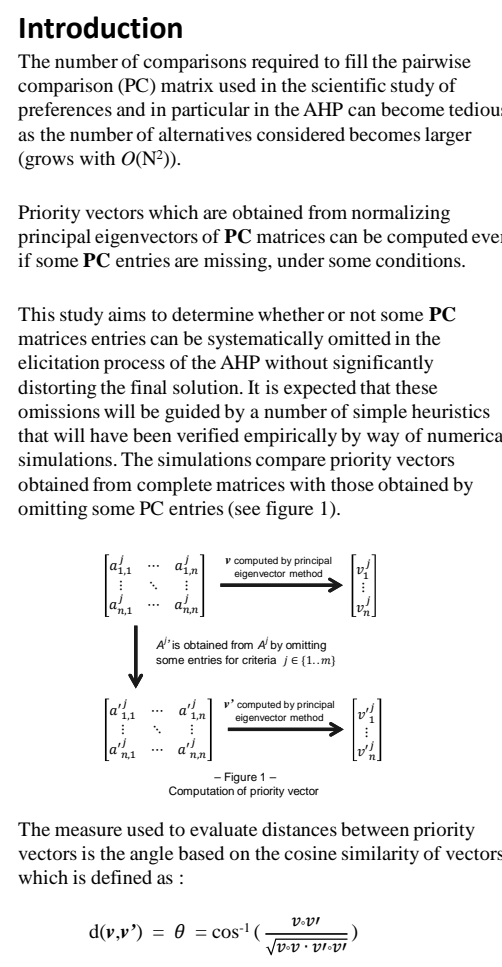

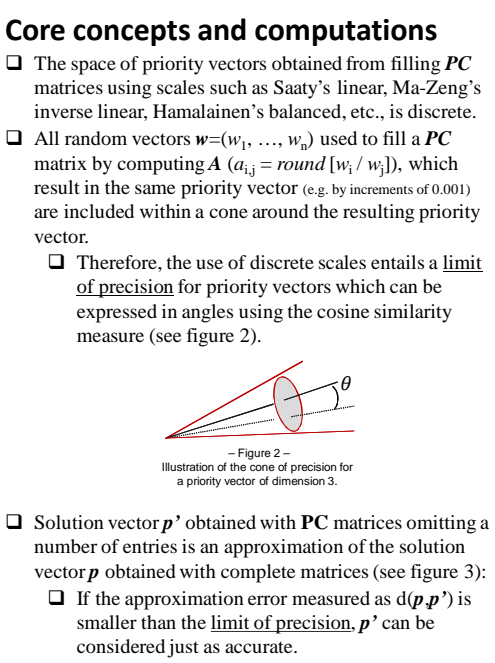

J Solution vector $\boldsymbol{p}$ ' obtained with $\mathbf{P C}$ matrices omitting a number of entries is an approximation of the solution vector $\boldsymbol{p}$ obtained with complete matrices (see figure 3 ):

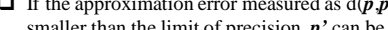
considered just as accurate.

\section{Preliminary Results}

Preliminary tests (as of this submission deadline - April 18, 2016) have

show :

That priority vectors obtained from matrices of sizes $5 \times 5$ to 15 X 15 have a limit of precision angle of approximately

That overall solutions with models of 6 to 8 alternatives and 4 to 8 criteria with incomplete matrices using a simp provide result vectors within less of 5 degrees from

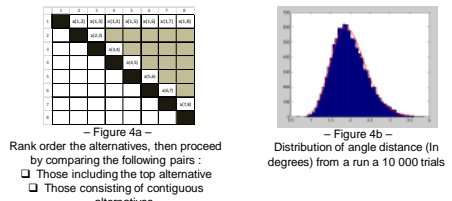

Mixed Integer Nonlinear Programming models have shown that the minimum numbers of entries required to approximate (wit

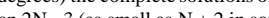

$\checkmark$ Further tests are being conducted :

With other preference scales (Inverse Linear, Balanced,..) To determine and evaluate other heuristics that could provide approximation with a lesser number of entries To determine the extent to which approximations are

Dns under which the heuristics can be

\section{Conclusions}

Preliminary results point towards the affirmation that the within limits of precision grow with $O(\mathrm{~N})$ (see table 1)

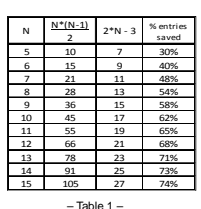

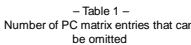

Further testing will help specify the conditions under which the heuristics could provide a significant reduction of effort in the elicitsion

\section{Literature references}

Prise Comparisons in the Analytic hierarchy Process", Mathematica Modelling, 9(11), 837-848.

Zahir, S., 1999, "Geometry of decision making and the vector space formulation of the analytic hierarchy process", European Journal of Operational Research, 112(2), 373-396
Ishizaka A., Labib A., 2011, "Review of the main developments in the analytic hierarchy process", Exper

Bozóki, S., Dezsō, L., Poesz, A., Temesi, J., 2013, “Analysis of 